\title{
Early and long-term outcomes of complete revascularization with percutaneous coronary intervention in patients with multivessel coronary artery disease presenting with non-ST-segment elevation acute coronary syndromes
}

\author{
Michał Hawranek ${ }^{1}$, Piotr Desperak ${ }^{1}$, Paweł Gąsior ${ }^{1}$, Aneta Desperak ${ }^{1}$, Andrzej Lekston ${ }^{1}$, Mariusz Gąsior ${ }^{2}$ \\ ${ }^{1} 3^{\text {rd }}$ Department of Cardiology, School of Medicine with the Division of Dentistry in Zabrze, Medical University of Silesia, Katowice, Silesian \\ Centre for Heart Disease in Zabrze, Poland \\ ${ }^{2} 3^{\text {rd }}$ Department of Cardiology, Medical University of Silesia in Katowice, School of Medicine in Katowice, Poland
}

Adv Interv Cardiol 2018; 14, 1 (51): 32-41

DOI: https://doi.org/10.5114/aic.2018.74353

\begin{abstract}
A bstract
Introduction: The clinical significance of complete revascularization with percutaneous coronary intervention (CR-PCI) in patients with non-ST-segment acute coronary syndrome (NSTE-ACS) remains uncertain.

Aim: To evaluate the impact of CR-PCI during index hospitalization on short and long-term incidence of death and composite endpoint among patients with multivessel coronary artery disease (CAD) presenting with NSTE-ACS.

Material and methods: We analyzed consecutive data of 1,592 patients with multivessel CAD from 2006 to 2014. Patients with prior coronary artery bypass grafting (CABG), cardiogenic shock, treated conservatively or with CABG and scheduled for planned CABG or PCl after discharge were excluded. The 30-day and 12-month composite endpoint was defined as all-cause death, nonfatal myocardial infarction (MI) or ACS-driven unplanned revascularization. Six hundred and ninety-five patients were divided into 2 groups: $\mathrm{CR}-\mathrm{PCI}(n=137)$ (CR-PCI during index hospitalization) and IR-PCI $(n=558)$ (incomplete revascularization).

Results: Incidence of composite endpoint (3.6\% vs. $10.2 \%$; $\mathrm{HR}=0.31 ; 95 \% \mathrm{Cl}: 0.12-0.87 ; p=0.025)$ and death $(0.7 \%$ vs. $5.7 \%$, $\mathrm{HR}=0.11 ; 95 \% \mathrm{Cl}: 0.02-0.93 ; p=0.043)$ at 30 days was lower in CR-PCI than in IR-PCI. At 12-month follow-up occurrence of composite endpoint was lower in CR-PCI (14.7\%) than in IR-PCI $(27.4 \%, p=0.0037)$. Multivariate analysis confirmed that CR PCI was associated with a reduction in 12 -month composite endpoint $(\mathrm{HR}=0.56 ; 95 \% \mathrm{Cl}: 0.31-0.99 ; p=0.046)$. The 12 -month mortality was lower in $\mathrm{CR}-\mathrm{PCl}(7.4 \%$ vs. $14.8 \% ; p=0.031)$, but it was not confirmed in the multivariate analysis.

Conclusions: In patients with multivessel CAD and NSTE-ACS, CR-PCI during index hospitalization was independently associated with improved early and long-term prognosis without significant differences in periprocedural outcomes in comparison to IR-PCI.
\end{abstract}

Key words: coronary artery revascularization, coronary percutaneous intervention, incomplete coronary revascularization, non-ST segment elevation myocardial infarction.

\section{Introduction}

Multivessel coronary artery disease (CAD) is found to be present in approximately $40-70 \%$ of patients presenting with non-ST-segment elevation acute coronary syndromes (NSTE-ACS) undergoing coronary angiography [1-7]. Presence of multivessel CAD is associated with worse clinical outcomes when compared to single vessel CAD $[7,8]$. After determining the artery responsible for NSTE-ACS manifestation, in most patients the firstchoice procedure is culprit-lesion percutaneous coronary intervention (PCI) [1, 2]. Nonetheless, in multivessel CAD, revascularization of vessels not directly responsible for acute myocardial ischemia still remains a controversial issue. It seems that patients with NSTE-ACS and multivessel CAD who underwent complete revascularization with percutaneous coronary intervention (CR-PCI) during index hospitalization could gain potential benefits over incomplete revascularization (IR-PCI) in terms of longterm prognosis [9-13]. It is well documented that the plaque disruption or frank rupture, presumably related to a heightened inflammatory milieu, may not be limited to the single artery but may involve other territories in the coronary artery system $[14,15]$. Moreover, more complete intervention may reduce adverse cardiovas-

\section{Corresponding author:}

Piotr Desperak MD, $3^{\text {rd }}$ Chair and Department of Cardiology, Medical University of Silesia in Katowice, School of Medicine with the Division of Dentistry in Zabrze, Silesian Center for Heart Diseases, 2 Szpitalna St, 41-800 Zabrze, Poland, e-mail: piotr.desperak@op.pl

Received: 21.10.2017, accepted: 30.01.2018. 
cular events, in particular re-hospitalization and future revascularization $[9,10,16-23]$. Current guidelines recommend that the choice of management in the mentioned situation should be individualized, dependent on the general condition of the patient, characteristics of lesions and severity of myocardial damage [1, 2]. Although complete coronary revascularization may provide benefits, $\mathrm{CR}-\mathrm{PCl}$ is frequently limited by anatomic conditions such as total chronic occlusions or severe CAD. There is lack of contemporary data regarding feasibility of CR-PCI during the index hospital stay.

\section{Aim}

We decided to perform a comparative analysis of CR$\mathrm{PCl}$ and IR-PCI during index hospitalization with respect to early (30-day) and long-term (12-month) prognosis among patients with multivessel CAD presenting with NSTE-ACS.

\section{Material and methods}

\section{Study design}

An analysis of consecutive data of 1,592 patients with NSTE-ACS and multivessel CAD from 1 January, 2006 to 31 December, 2014 was comprehensively undertaken. The exclusion criteria were: prior coronary artery bypass grafting (CABG), occurrence of cardiogenic shock, treatment with $C A B G$ in the course of initial hospitalization and scheduled to planned revascularization (CABG or $\mathrm{PCl}$ ) after discharge. In the further analysis, patients were divided into 2 groups depending on completeness of revascularization during the index hospital stay: (I) CR$\mathrm{PCl}$ group - complete revascularization following $\mathrm{PCl}$; and (II) IR-PCI group - incomplete revascularization.

All enrolled patients underwent $\mathrm{PCl}$ using standard techniques. All interventional strategies, including completeness of revascularization, the use of stents, choice of stent type, and periprocedural anti-thrombin and antiplatelet therapy, were at the operator's or heart team's discretion. Before and after the intervention, pharmacological treatment recommended by the European Society of Cardiology (ESC) was introduced [1, 2]. The demographic, clinical and angiographic data collected in the course of the index hospitalization were retrieved from the prospectively recorded Institutional Electronic Database. The post-hospitalization data with the accompanying exact dates of death, myocardial infarction (MI) and acute coronary syndrome (ACS) driven unplanned revascularization were obtained from the official National Health Fund records. The vital status at 12 months was available for all of the patients.

The study was granted permission from the Institutional Review Board and University Bioethics Committee, and is in accordance with the ethical standards laid down in the 1964 Declaration of Helsinki and its later amendments.
Patients with confirmed diagnosis of NSTE-ACS based on clinical symptoms and electrocardiography were enrolled in the registry, and then classified as having unstable angina or non-ST-segment elevation myocardial infarction based on measured levels of markers of myocardial necrosis [1, 24]. Multivessel CAD was defined as hemodynamically significant stenosis in the left main $(L M)$ or in $\geq 2$ major epicardial territories or in their major branches (left anterior descending (LAD), left circumflex (LCX) or right coronary artery system (RCA)) with a diameter $\geq 2.0 \mathrm{~mm}$ as determined by visual assessment. Lesions with $\geq 50 \%$ diameter stenosis in the $L M$ or proximal segment of the LAD and $\geq 70 \%$ diameter stenosis in other segments were considered as hemodynamically significant. A coronary artery was considered to be a culprit if one of the following criteria was present: definite or suspect thrombus, ruptured or ulcerated plaque, presence of thrombolysis in myocardial infarction (TIMI) flow grade $\leq 2$, and tight stenosis $\geq 70 \%$ consistent with non-invasive ischemia tests. Angiographic success was defined as the achievement of a minimum stenosis diameter reduction to $<20 \%$ in the presence of TIMI flow 3 grade [25].

The targeted approach in the study population was complete functional revascularization of coronary arteries. Myocardial viability was assessed using single-photon emission computed tomography or stress echocardiography. The decision on revascularization of non-culprit lesions was at the operator's or team's discretion. Due to observational nature of the study, we adopted the anatomic definition of CR-PCI as the successful invasive treatment of all significant stenoses in major epicardial coronary arteries or their side branches with a diameter $\geq 2.0 \mathrm{~mm}$ during initial hospitalization caused by NSTEACS irrespective of the function or viability of relevant myocardium $[26,27]$.

\section{Endpoints and definitions}

The primary outcome measure included the occurrence of the composite endpoint defined as the composite rate of (1) death, (2) nonfatal recurrent myocardial infarction or (3) ACS-driven unplanned revascularization in a 30-day and 12-month observation period. Death was considered as all-cause death. Non-fatal MI was defined as an ischemic event that met the ESC/American College of Cardiology criteria for myocardial infarction and clearly clinically separate from the baseline ACS at the time of admission [24]. Acute coronary syndrome-driven repeat revascularization was defined as additional, unplanned angioplasty or $\mathrm{CABG}$, performed as an urgent procedure because of acute ischemic symptoms [28].

The secondary outcome measures were singular components of the primary end point, the incidence of in-hospital events (death, non-fatal MI, target vessel revascularization (TVR), contrast-induced nephropathy (CIN), and bleeding event) and the independent factors 
influencing the performance and achieving of CR-PCI. The CIN was defined as impaired renal function on the basis of relative $\geq 25 \%$ or absolute $\geq 44 \mu \mathrm{mol} / /$ increase of creatinine concentration in blood serum up to 3 days after the first or following coronary angiography and absence of an alternative explanation of renal dysfunction [29]. Bleeding was defined as clinically overt bleeding: i) with an ensuing decrease in hemoglobin to below $5 \mathrm{~g} / \mathrm{dl}(3.1 \mathrm{mmol} / \mathrm{l})$ or absolute decrease of hematocrit by more than $15 \%$; or ii) resulting in hemodynamic disorders; or iii) requiring blood transfusion.

\section{Statistical analysis}

The statistical analysis included comparison of baseline, angiographic and in-hospital characteristics, and occurrence of the composite endpoint and its components in the 30-day and 12-month follow-up period. Analyzed variables are expressed as numbers and percentages. Continuous variables were summarized using the arithmetic mean with standard deviation (SD) for data following a normal distribution or median with quartiles 1 and 3 (Q1-Q3) for data following a distribution other than normal. Student's $t$-test was performed for comparison of continuous parameters with a normal distribution, whereas the Mann-Whitney $U$ test was performed for parameters with a distribution other than normal. Normality of the distribution was verified using the Shapiro-Wilk test. Categorical variables were summarized using frequency tables and were compared using the $\chi^{2}$ Pearson test. Composite endpoint, all-cause mortality, non-fatal $\mathrm{MI}$ and revascularization caused by ACS in the 12-month observation period for all patients were analyzed using the Kaplan-Meier method with the

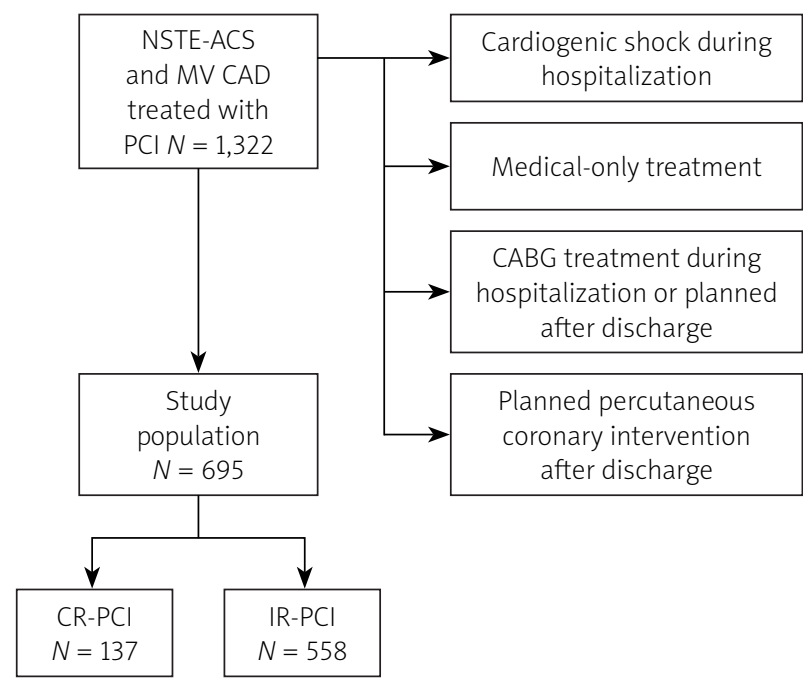

Figure 1. Study design

$C A B G$ - coronary artery bypass graft, $C A D$ - coronary artery disease, $C R-P C l$ - complete revascularization with percutaneous coronary intervention, $I R-P C I$ - incomplete revascularization with percutaneous coronary intervention, NSTE-ACS - non-ST-segment elevation acute coronary syndromes, $\mathrm{PCl}$ - percutaneous coronary intervention. log-rank test. Factors affecting 30-day and 12-month outcomes were analyzed using a Cox proportional hazards model. In the analysis we included all of the covariates which were significant in the univariate analysis. Furthermore, to evaluate factors affecting the ability to achieve the $\mathrm{CR}-\mathrm{PCl}$, a logistic regression model was used. Candidate variables were all statistically significant parameters from baseline and angiographic characteristics. In the analysis we included all of the covariates which were significant in the univariate analysis. Results of the multivariate analyses were summarized as the odds ratio (OR) or hazard ratio (HR) with $95 \%$ confidence interval $(\mathrm{Cl})$. For all analyses a 2-tailed $p$-value $\leq 0.05$ was considered as significant. The Statistica 10 software (StatSoft Inc., Tulsa, Oklahoma) and MedCalc 12.2.1 (MedCalc Software, Mariakerke, Belgium) were used for all calculations.

\section{Results}

The study population is presented in Figure 1. Among 695 patients with multivessel CAD presenting with NSTE-ACS enrolled in the study, complete revascularization was achieved in 137 patients (CR-PCI group), while the remaining 558 patients did not undergo complete revascularization (IR-PCI group) in the course of the initial hospitalization. In the CR-PCI group, complete revascularization was achieved in 81 cases during the index procedure, whereas it was achieved in 47 patients with multi-stage $\mathrm{PCl}$. The clinical characteristics of the study population stratified by performance of CR-PCI or IR-PCI are summarized in Table I. The analysis of coronary angiography and procedural parameters is presented in Table II. The procedural success of intervention in the culprit vessel and all treated lesions in the CR-PCI group was $100 \%$ according to the adopted definition of completeness of revascularization. Among patients from the CR-PCl group, calcium antagonists (22.9 vs. $37.5 \%$; $p=0.0035)$ and diuretics (32.4 vs. 42.4; $p=0.051$ ) were less often recommended upon discharge from hospital. Moreover, there were no differences in drug therapies between groups.

At the index hospital stay, no significant differences in studied groups regarding incidence of adverse events, in particular in rates of death (1.5\% vs. $3.8 \% ; p=0.17)$ and non-fatal MI (0.7\% vs. $1.8 \% ; p=0.37)$, were observed. Moreover, the occurrence of bleeding (4.4\% vs. $3.8 \%$; $p=0.15)$ and CIN (12.4\% vs. 9.3\%; $p=0.28)$ was similar in both groups. However, at 30 days, independently lower occurrence of composite endpoint (3.6\% vs. 10.2\%; $\mathrm{HR}=0.31 ; 95 \% \mathrm{Cl}: 0.12-0.87 ; p=0.025)$ and all-cause death $(0.7 \%$ vs. $5.7 \%$; $\mathrm{HR}=0.11 ; 95 \% \mathrm{Cl}: 0.02-0.93$; $p=0.014)$ in CR-PCI than IR-PCI was found.

During the 12-month observation period, a significant difference in the occurrence of composite endpoint was also maintained: $16.1 \%$ for the CR-PCI group and $27.9 \%$ 
Table I. Baseline characteristics of patients according to performance of complete revascularization with percutaneous coronary intervention in the study groups

\begin{tabular}{|c|c|c|c|}
\hline \multirow[t]{2}{*}{ Variable } & \multicolumn{2}{|c|}{ CR-PCI } & \multirow[t]{2}{*}{$P$-value } \\
\hline & $\begin{array}{c}\text { Yes } \\
n=137(19.7 \%) \\
\end{array}$ & $\begin{array}{c}\text { No } \\
n=558(80.3 \%)\end{array}$ & \\
\hline Age, mean \pm SD [years] & $65.7 \pm 11.3$ & $68.4 \pm 10.5$ & 0.013 \\
\hline Final NSTEMI diagnosis (\%) & 70.1 & 64.5 & 0.22 \\
\hline Males (\%) & 67.1 & 67.4 & 0.96 \\
\hline Arterial hypertension (\%) & 76.6 & 76.0 & 0.87 \\
\hline Prior MI (\%) & 27.6 & 47.6 & $<0.0001$ \\
\hline Prior PCI (\%) & 28.4 & 36.9 & 0.064 \\
\hline Atrial fibrillation (\%) & 11.2 & 11.7 & 0.86 \\
\hline Peripheral artery disease (\%) & 11.9 & 15.3 & 0.32 \\
\hline Prior stroke (\%) & 5.2 & 8.7 & 0.19 \\
\hline Diabetes mellitus (\%) & 30.9 & 41.5 & 0.023 \\
\hline Insulin treatment (\%) & 11.9 & 20.2 & 0.027 \\
\hline Dyslipidemia (\%) & 71.8 & 66.2 & 0.21 \\
\hline Obesity (\%) & 23.9 & 28.3 & 0.30 \\
\hline COPD (\%) & 1.5 & 7.6 & 0.0097 \\
\hline History of cigarette smoking (\%) & 48.9 & 39.3 & 0.043 \\
\hline Familial history of MI (\%) & 23.1 & 21.7 & 0.71 \\
\hline Chest pain (\%) & 92.5 & 90.8 & 0.52 \\
\hline Killip class III (\%) & 1.5 & 3.6 & 0.21 \\
\hline Heart rate, mean \pm SD [bpm] & $77 \pm 16$ & $78 \pm 17$ & 0.35 \\
\hline $\mathrm{SBP}$, mean $\pm \mathrm{SD}[\mathrm{mm} \mathrm{Hg}]$ & $148 \pm 26$ & $146 \pm 30$ & 0.51 \\
\hline $\mathrm{DBP}$, mean $\pm \mathrm{SD}[\mathrm{mm} \mathrm{Hg}]$ & $86 \pm 17$ & $86 \pm 17$ & 0.89 \\
\hline $\mathrm{BMI}$, mean $\pm \mathrm{SD}\left[\mathrm{kg} / \mathrm{m}^{2}\right]$ & $28.5 \pm 4.6$ & $28.8 \pm 5.2$ & 0.61 \\
\hline ST-segment deviation (\%) & 41.1 & 43.3 & 0.68 \\
\hline LBBB (\%) & 7.5 & 7.0 & 0.87 \\
\hline RBBB (\%) & 1.9 & 6.3 & 0.068 \\
\hline CK-MB [ng/ml] (Q1-Q3) & $10.1(3.5-39.6)$ & $7.3(3.3-36.0)$ & 0.13 \\
\hline TcT [ng/ml] (Q1-Q3) & $0.1(0.0-0.5)$ & $0.1(0.0-0.5)$ & 0.79 \\
\hline $\mathrm{TC}[\mathrm{mmol} / \mathrm{l}](\mathrm{Q} 1-\mathrm{Q} 3)$ & $5.1(4.1-6.0)$ & $5.0(4.0-5.9)$ & 0.48 \\
\hline LDL [mmol/l] (Q1-Q3) & $3.0(2.1-4.0)$ & $3.0(2.2-3.8)$ & 0.68 \\
\hline WBC [× 103/ul] (Q1-Q3) & $8.1(6.9-10.5)$ & $8.6(6.9-11.4)$ & 0.23 \\
\hline Hemoglobin, mean \pm SD [mmol/l] & $8.5 \pm 1.2$ & $8.4 \pm 1.1$ & 0.33 \\
\hline Glucose [mmol/l] (Q1-Q3) & $6.3(5.5-8.1)$ & $6.7(5.5-8.7)$ & 0.32 \\
\hline Serum creatinine $[\mu \mathrm{mol} / \mathrm{l}](\mathrm{Q} 1-\mathrm{Q} 3)$ & $80(69-94)$ & $88(73-113)$ & 0.014 \\
\hline GFR $\left[\mathrm{ml} / \mathrm{min} / 1.73 \mathrm{~m}^{2}\right]$ (Q1-Q3) & $81(67-98)$ & $72(51-92)$ & 0.0038 \\
\hline $\mathrm{GFR} \leq 60 \mathrm{ml} / \mathrm{min} / 1.73 \mathrm{~m}^{2}$ & 17.8 & 35.2 & 0.0008 \\
\hline LVEF (\%) (Q1-Q3) & $48(40-52)$ & $42(33-50)$ & $<0.0001$ \\
\hline LVEF $\leq 35 \%(\%)$ & 15.4 & 31.3 & 0.0003 \\
\hline GRACE Score, mean \pm SD [points] & $120 \pm 31$ & $128 \pm 30$ & 0.0083 \\
\hline
\end{tabular}

$B M I$ - body mass index, CK-MB - creatine kinase muscle-brain type, COPD - chronic obstructive pulmonary disease, $C R-P C I$ - complete revascularization with percutaneous coronary intervention, DBP - diastolic blood pressure, GFR - glomerular filtration rate, LBBB - left bundle branch block, LDL - low-density lipoprotein, $M I$ - myocardial infarction, NSTEMI-non-ST-segment elevation myocardial infarction, $P C I$ - percutaneous coronary intervention, RBBB - right bundle branch block, SBP - systolic blood pressure, SD - standard deviation, Q1-Q3 - quartile 1 and quartile 3, TC - total cholesterol, TCT - cardiac troponin T, WBC - white blood cells. 
Table II. Angiographic and procedural characteristic of patients according to performance of complete revascularization with percutaneous coronary intervention in the study groups

\begin{tabular}{|c|c|c|c|}
\hline \multirow[t]{2}{*}{ Variable } & \multicolumn{2}{|c|}{ CR-PCI } & \multirow[t]{2}{*}{$P$-value } \\
\hline & $\begin{array}{c}\text { Yes } \\
n=137(19.7 \%)\end{array}$ & $\begin{array}{c}\text { No } \\
n=558(80.3 \%)\end{array}$ & \\
\hline Femoral access (\%) & 84.7 & 88.6 & 0.19 \\
\hline Radial access (\%) & 15.3 & 11.4 & 0.22 \\
\hline 2-vessel CAD (\%) & 89.0 & 57.3 & $<0.0001$ \\
\hline 3-vessel CAD (\%) & 11.0 & 42.6 & $<0.0001$ \\
\hline LM CAD (\%) & 11.7 & 9.1 & 0.37 \\
\hline CTO of non-culprit vessel (\%) & 0.0 & 69.7 & $<0.0001$ \\
\hline $\mathrm{PCl}$ ad hoc (\%) & 95.6 & 88.7 & 0.015 \\
\hline IRA LM (\%) & 8.9 & 4.6 & 0.049 \\
\hline IRA LAD (\%) & 31.1 & 34.5 & 0.46 \\
\hline IRA CX (\%) & 34.1 & 31.2 & 0.52 \\
\hline IRA RCA (\%) & 25.9 & 29.7 & 0.38 \\
\hline Restenotic lesion (\%) & 8.1 & 10.8 & 0.36 \\
\hline Bifurcation (\%) & 21.5 & 22.0 & 0.89 \\
\hline Baseline TIMI flow grade 0-1 (\%) & 16.3 & 22.0 & 0.14 \\
\hline Stent placement (\%) & 93.3 & 86.8 & 0.036 \\
\hline Balloon predilatation (\%) & 59.3 & 62.9 & 0.43 \\
\hline Balloon postdilatation (\%) & 15.6 & 15.1 & 0.88 \\
\hline DES (\%) & 40.0 & 31.9 & 0.075 \\
\hline Procedural glycoprotein IIb/IIla inhibitor (\%) & 5.9 & 7.3 & 0.56 \\
\hline Dissection (\%) & 4.4 & 7.0 & 0.28 \\
\hline No/slow reflow (\%) & 1.5 & 0.9 & 0.56 \\
\hline Final TIMI flow grade 3 after $\mathrm{PCI}(\%)$ & 100.0 & 90.6 & 0.0022 \\
\hline Angiographic success of PCI IRA (\%) & 100.0 & 88.3 & 0.0003 \\
\hline $\mathrm{PCl}$ of additional artery during hospitalization (\%): & 91.1 & 19.2 & $<0.0001$ \\
\hline One-staged & 59.1 & 14.9 & $<0.0001$ \\
\hline Multi-staged & 34.3 & 4.7 & $<0.0001$ \\
\hline $\begin{array}{l}\text { Angiographic success of all treated lesions during } \\
\text { hospitalization (\%) }\end{array}$ & 100.0 & 86.0 & $<0.0001$ \\
\hline
\end{tabular}

CAD - coronary artery disease, CR-PCI - complete revascularization with percutaneous coronary intervention, CTO - chronic total occlusion, CX - circumflex artery, $D E S$ - drug-eluting stent, IRA - ischemic-related artery, LAD - left anterior descending artery, LM - left main, PCI - percutaneous coronary intervention, RCA - right coronary artery, TIMI - thrombolysis in myocardial infarction.

for the IR-PCI group (Figure $2 \mathrm{~A} ; p$ log-rank = 0.0036). In the multivariate analysis of the entire study population, the performance of CR-PCI independently decreased the risk of 12-month composite endpoint (Table III; $\mathrm{HR}=0.56 ; 95 \% \mathrm{Cl}: 0.31-0.99 ; p=0.046)$. Others independent factors influencing the 12-month composite endpoint were presented in Table IV. A significant effect of completeness of revascularization on the occurrence of all-cause mortality in the 12-month observation period was observed (Figure 2 B; $8.0 \%$ vs. $15.8 \%$; $p$ log-rank $=0.018)$, but it was not confirmed in the multivariate analysis ( $\mathrm{HR}=0.78 ; 95 \% \mathrm{Cl}: 0.34-1.80 ; p=0.56)$. There were no statistical differences observed at 12 months for rates of non-fatal MI (Figure 2 C; $5.1 \%$ vs. 9.3\%; $p$ log-rank $=0.11$ ) and ACS-driven unplanned revascularization (Figure 2 D; 9.5\% vs. 9.9\%; $p$ log-rank =0.88). Independent predictors of incomplete revascularization were occurrence of chronic total occlusion (CTO), higher mean age, higher GRACE risk score and lower incidence of 2-vessel CAD in study patients (Table V).

\section{Discussion}

In several studies analyzing patients with NSTE-ACS and multivessel CAD, multivessel PCI during index hospitalization versus multistage procedures is one of the main raised concerns [16-23]. In contrast to stable angi- 
A

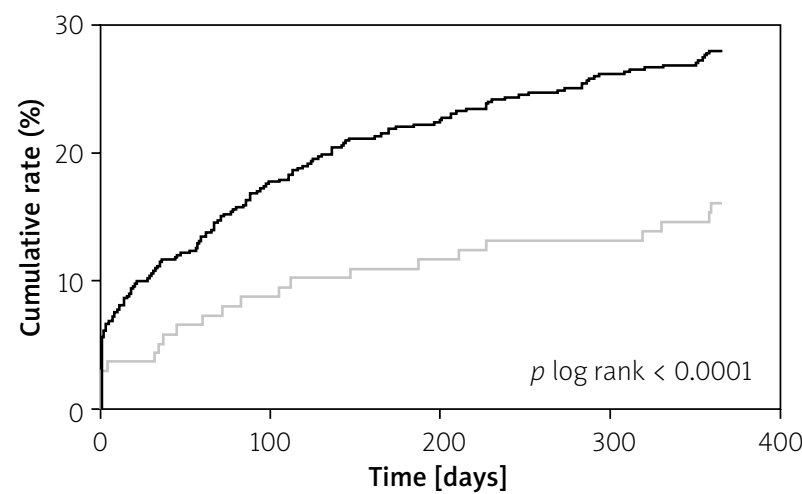

No. at risk, $N$

$\begin{array}{lccr}\text { CR-PCI 137 } & 124 & 119 & 117 \\ \text { IR-PCI } 558 & 458 & 431 & 411 \\ & & \text { CR-PCl group } & - \text { IR-PCI group }\end{array}$

C

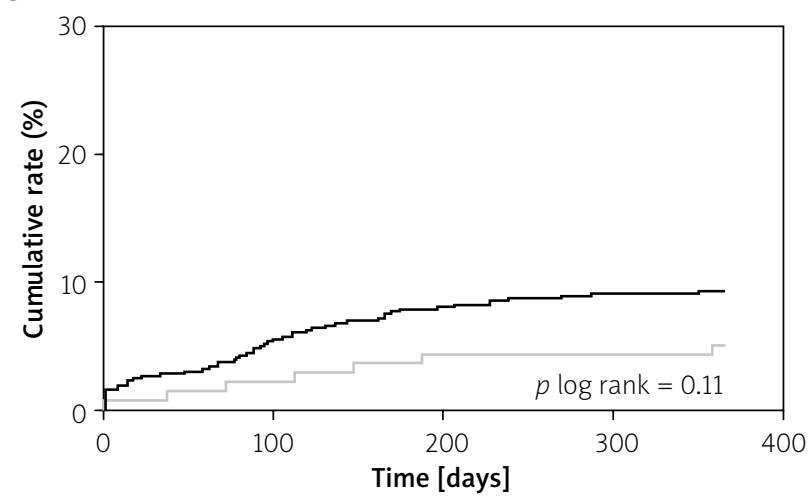

No. at risk, $N$

CR-PCl 137

IR-PCI 558
B

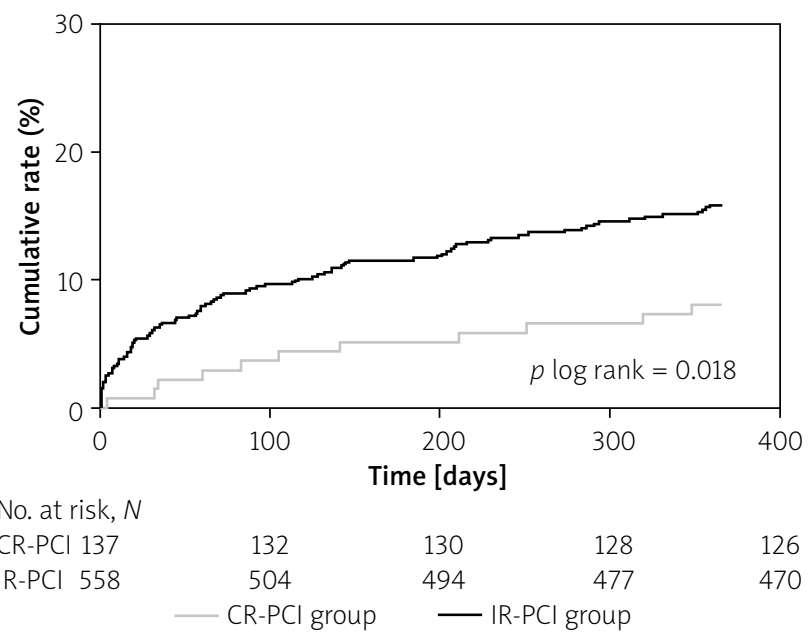

D

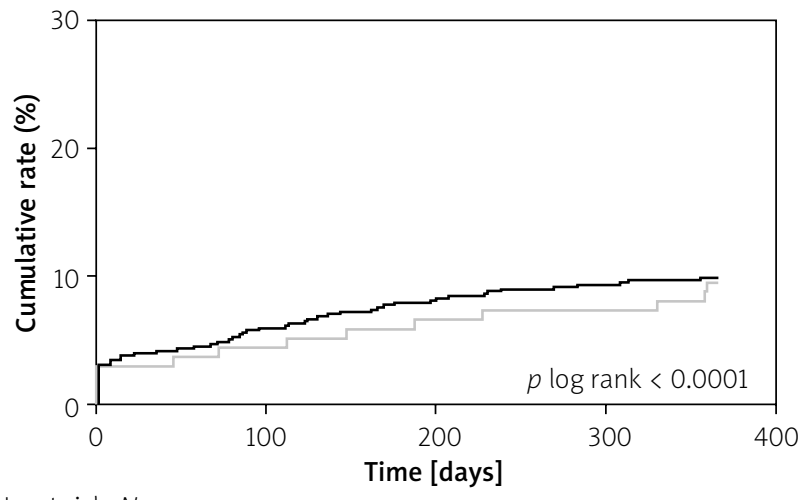

No. at risk, $N$

CR-PCl 137

IR-PCI 558

Figure 2. Kaplan-Meier survival curves for 12-month rates of major adverse cardiac events (A), death for any cause (B), non-fatal myocardial infarction (C) and revascularization caused by acute coronary syndrome (D) according to performance of complete revascularization with percutaneous coronary intervention in the study groups

$C R-P C I$ - complete revascularization with percutaneous coronary intervention, IR-PCI - incomplete revascularization with percutaneous coronary intervention, Ml-myocardial infarction.

na (SA) patients [11-13, 30, 31], only a few studies have revolved around completeness of revascularization of significantly narrowed segments in NSTE-ACS patients $[9,10]$. Summarizing the presented results we concluded that: 1 ) in less than $20 \%$ of the study population CR-PCI was achieved, 2) the analysis did not show any statistically significant differences in periprocedural and in-hospital outcomes and 3) possibility of performing the CR-PCI strategy was associated with a $69 \%$ reduction in occurrence of the composite endpoint at 30 days and a $44 \%$ reduction of the composite endpoint in the 12-month observation period in comparison to IR-PCI. Moreover, 4) severity of coronary artery obstruction, occurrence of CTO assessed by coronary angiography, GRACE risk score and mean age of treated patients were important factors af- fecting the decision of implementation of CR-PCI during the index hospital stay.

As previously noted, there is high volatility in the criteria adopted to define the complete revascularization of coronary arteries [11-13, 26, 30, 31]. Currently in clinical practice complete functional revascularization seems to be the most reasonable and the most appropriate approach in the majority of patients [27, 32]. However, as well as in the majority of cited studies, due to the retrospective nature of our analysis, the definition of CR-PCI was judged on an anatomical basis, owing to the unavailability of data on myocardial viability or fractional flow reserve revaluation of stenosed coronary segments. Another important aspect about considerations of the CR-PCI strategy is the selection of the 
Table III. Unadjusted and adjusted hazard ratios for occurrence of 30-day and 12-month outcomes of patients according to performance of complete revascularization with percutaneous coronary intervention

\begin{tabular}{|c|c|c|c|c|c|c|}
\hline Event (CR-PCI vs. IR-PCI) & Unadjusted HR & $95 \% \mathrm{Cl}$ & $P$-value & Adjusted HR* & $95 \% \mathrm{Cl}$ & $P$-value \\
\hline 30-day composite endpoint: & 0.35 & $0.14-0.87$ & 0.024 & 0.31 & $0.12-0.87$ & 0.025 \\
\hline Death & 0.13 & $0.02-0.91$ & 0.040 & 0.11 & $0.02-0.93$ & 0.043 \\
\hline Non-fatal MI & 0.26 & $0.04-1.90$ & 0.18 & - & - & - \\
\hline ACS-driven revascularization & 0.78 & $0.27-2.26$ & 0.64 & - & - & - \\
\hline 12-month composite endpoint (\%): & 0.50 & $0.30-0.81$ & 0.0054 & 0.56 & $0.31-0.99$ & 0.046 \\
\hline Death & 0.47 & $0.24-0.95$ & 0.034 & 0.78 & $0.34-1.80$ & 0.56 \\
\hline Non-fatal MI & 0.48 & $0.20-1.13$ & 0.095 & - & - & - \\
\hline ACS-driven revascularization & 0.74 & $0.36-1.50$ & 0.40 & - & - & - \\
\hline
\end{tabular}

*Candidate variables were following parameters: 3-vessel coronary artery disease, absence of chest pain on admission, age, atrial fibrillation, body mass index, chronic obstructive pulmonary disease, chronic total occlusion of non-culprit vessel, creatine kinase muscle-brain type on admission, CR-PCI, diastolic blood pressure on admission, female gender, final non-ST-segment elevation myocardial infarction diagnosis, glucose on admission, hemoglobin on admission, heart rate on admission, insulin-treatment diabetes mellitus, left bundle branch block, left main coronary artery disease, left ventricular ejection fraction, percutaneous coronary intervention of right coronary artery, peripheral arteries disease, prior myocardial infarction, serum creatinine on admission, success of percutaneous coronary intervention in culprit vessel, systolic blood pressure on admission, ST deviations on admission, white blood cells on admission. ACS - acute coronary syndrome, $\mathrm{Cl}$-confidence interval, $C R-P C I$ - complete revascularization with percutaneous coronary intervention, $\mathrm{HR}$ - hazard ratio, IR-PCI-incomplete revascularization with percutaneous coronary intervention, $\mathrm{MI}$ - myocardial infarction.

Table IV. Multivariate analysis for occurrence of 12-month composite endpoint

\begin{tabular}{|c|c|c|c|}
\hline Variable & HR & $95 \% \mathrm{Cl}$ & $P$-value \\
\hline Serum creatinine at admission (per 10 mmol/l more) & 1.02 & $1.01-1.04$ & 0.0080 \\
\hline Left ventricular ejection fraction (per 1\% more) & 0.98 & $0.97-1.00$ & 0.022 \\
\hline Diabetes mellitus & 1.43 & $1.05-1.95$ & 0.024 \\
\hline CR-PCl & 0.56 & $0.31-0.99$ & 0.046 \\
\hline $\mathrm{PCl}$ of left main & 1.95 & $1.01-3.80$ & 0.049 \\
\hline Prior myocardial infarction & 1.31 & $0.97-1.78$ & 0.066 \\
\hline Body mass index (per 1 kg/m² more) & 0.96 & $0.93-1.00$ & 0.075 \\
\hline Hemoglobin at admission (per $1 \mathrm{mmol} / \mathrm{l}$ more) & 0.87 & $0.74-1.02$ & 0.079 \\
\hline Diagnosis of myocardial infarction & 1.32 & $0.95-1.83$ & 0.095 \\
\hline Peripheral artery disease & 1.38 & $0.94-2.03$ & 0.11 \\
\hline NYHA class IV at admission & 1.51 & $0.77-2.97$ & 0.23 \\
\hline Age (per 1 year more) & 0.99 & $0.98-1.01$ & 0.32 \\
\hline Pulmonary edema & 0.84 & $0.45-1.59$ & 0.60 \\
\hline Heart rate (per 10 bpm more) & 1.01 & $0.93-1.11$ & 0.64 \\
\hline 3-vessel coronary artery disease & 0.96 & $0.70-1.32$ & 0.80 \\
\hline
\end{tabular}

${ }^{*}$ Candidate variables were the following parameters: 3-vessel coronary artery disease, absence of chest pain on admission, age, atrial fibrillation, body mass index, chronic obstructive pulmonary disease, chronic total occlusion of non-culprit vessel, creatine kinase muscle-brain type on admission, CR-PCl, diastolic blood pressure on admission, female gender, final non-ST-segment elevation myocardial infarction diagnosis, glucose on admission, hemoglobin on admission, heart rate on admission, insulin-treatment diabetes mellitus, left bundle branch block, left main coronary artery disease, left ventricular ejection fraction, percutaneous coronary intervention of right coronary artery, peripheral artery disease, prior myocardial infarction, serum creatinine on admission, success of percutaneous coronary intervention in culprit vessel, systolic blood pressure on admission, ST deviations on admission, white blood cells on admission. CI-confidence interval, CR-PCI-complete revascularization with percutaneous coronary intervention, HR - hazard ratio, NYHA - New York Heart Association.

study group. In patients with cardiogenic shock, guidelines recommend complete revascularization of critically narrowed coronary arteries; therefore occurrence of cardiogenic shock may potentially affect the choice of applied treatment $[2,33]$. Patients with planned revascularization after discharge were excluded from the analysis in most studies $[18,23]$. In contrast to existing publications we included patients with left main 
Table V. Unadjusted and adjusted odds ratios in multivariate analysis of factors influencing the performance of complete revascularization with percutaneous coronary intervention

\begin{tabular}{lcccccc} 
Factor & Unadjusted HR & $\mathbf{9 5 \% ~ C l}$ & $\boldsymbol{P}$-value & Adjusted HR* & $95 \% \mathrm{Cl}$ & $P$-value \\
\hline Chronic total occlusion & 0.01 & $0.01-0.03$ & $<0.0001$ & 0.01 & $0.01-0.03$ & $<0.0001$ \\
\hline 2-vessel CAD & 6.05 & $3.45-10.61$ & $<0.0001$ & 4.73 & $2.18-10.28$ & 0.0001 \\
\hline Age (per 1 year more) & 0.97 & $0.96-0.99$ & 0.0091 & 0.95 & $0.92-0.99$ & 0.0071 \\
\hline GRACE score (per 10 points more) & 0.91 & $0.86-0.98$ & 0.0078 & 0.92 & $0.86-0.99$ & 0.0088 \\
\hline Creatinine (per 10 umol// more) & 0.94 & $0.89-0.99$ & 0.014 & 0.93 & $0.87-0.99$ & 0.036 \\
\hline COPD & 0.18 & $0.04-0.77$ & 0.020 & 0.36 & $0.08-1.83$ & 0.22 \\
\hline TC (per 1 mmol/l more) & 1.16 & $1.01-1.33$ & 0.036 & 1.13 & $0.92-1.38$ & 0.24 \\
\hline Prior MI & 0.42 & $0.28-0.64$ & $<0.0001$ & 0.69 & $0.33-1.41$ & 0.30 \\
\hline LVEF (per 1\% more) & 1.04 & $1.02-1.06$ & 0.0001 & 1.01 & $0.99-1.04$ & 0.32 \\
\hline Insulin-treatment diabetes mellitus & 0.53 & $0.31-0.94$ & 0.021 & 0.98 & $0.45-2.14$ & 0.96
\end{tabular}

Area under the ROC curve (AUC) 0.912; 95\% confidence interval 0.887 to 0.934 standard error $0.012 .{ }^{*}$ Candidate variables were parameters from baseline and angiographic characteristics (Tables I and II). CAD - coronary artery disease, CI-confidence interval, COPD - chronic obstructive pulmonary disease, LVEF-left ventricular ejection fraction, $\mathrm{MI}$ - myocardial infarction, TC - total cholesterol.

disease $[9,16,18,22]$ and CTO in a non-culprit lesion [16-18] for clear reflection of real-world patients [34]. As mentioned, according to adopted definition of CR$\mathrm{PCl}$, high diversity in inclusion and division criteria, and other differences in study methodology, direct comparisons with our results might be ambiguous.

Only in $19.7 \%$ of patients did operators perform CR$\mathrm{PCl}$ during the index hospital stay, of whom $12.9 \%$ underwent a one-staged and $6.8 \%$ a multi-staged procedure. Recent studies regarding NSTE-ACS patients reported CR$\mathrm{PCl}$ rates ranging from $47.0 \%$ according to Palmer et al. [9] to $63.3 \%$ in a subanalysis of the ACUITY trial [10] (cutoff point of hemodynamic significance $>50 \%$ diameter stenosis). A high percentage of achieved CR-PCI compared to our data was achieved most likely due to methodological aspects: the relatively small group $(n=151)$ and the intention-to-treat strategy in the study of Palmer et al., and enrollment of patients with single-vessel CAD in subanalysis of the ACUITY trial. In studies concerning multivessel procedures, the percentage of patients treated with multivessel $\mathrm{PCI}$ ranged between $21.3 \%$ and $61.7 \%$ [16-23]. In general, the reasons for the small proportion of patients who underwent complete revascularization are: 1) the operator's inability to achieve the CR-PCl caused by anatomical features and 2) the decision to selectively revascularize only the culprit vessel due to the patient's clinical condition. The results of our analysis confirm previous findings and suggest that patients with NSTE-ACS and multivessel diseases should be carefully assessed before discharge in terms of performing complete revascularization.

We did not find significant differences in occurrence of adverse cardiac events during index hospitalization depending on performed revascularization strategy, although at 30 days, significantly better early prognosis in the CR-PCl group was observed. Data from contemporary studies analyzing in-hospital and early outcomes of patients with multivessel CAD presenting with NSTE-ACS who underwent more complete revascularization in comparison with culprit-only $\mathrm{PCl}$ are controversial and ambiguous [9, 10, 16-23]. In contrast to our study, the analyses by Brener et al. and Bauer et al. showed that one-staged multivessel $\mathrm{PCl}$ was associated with more frequent occurrence of periprocedural myocardial infarction without any significant difference in in-hospital mortality [21, 22]. Also Hassanin et al. reported that multivessel compared to culprit-only PCI was unambiguously connected with worse prognosis in the in-hospital and 30-day observation period [20]. Other studies in the NSTE-ACS population did not reveal any other differences in early outcomes $[9,10,16-18,23]$. The comparable rates of hospital $\mathrm{MI}$, bleeding, and acute kidney injury in our analysis suggest that CR-PCl during initial hospitalization in selected patients is feasible and safe.

Our study shows improved long-term prognosis in patients undergoing $C R-P C I$ in comparison with $I R-P C I$. In the analyzed population, after considering typical clinical and angiographic factors, implementation of CR-PCI independently reduced incidence of the composite endpoint by $44 \%$ at the 12 -month follow-up. Palmer et al. demonstrated that achieving $\mathrm{CR}-\mathrm{PCl}$ is correlated with reduction in residual angina, repeated $\mathrm{PCl}$ and need for multiple antianginal therapies at roughly 10-month observation [9]. In the subanalysis of the ACUITY trial, the $\mathrm{IR}-\mathrm{PCl}$ strategy independently increased the percentage of major adverse events at one year in comparison to $\mathrm{CR}-\mathrm{PCl}$, which was mainly associated with higher rates of $\mathrm{MI}$ and repeated revascularization [10]. Other authors 
reported that multivessel $\mathrm{PCI}$ was associated with reduction of major adverse cardiac events due to decreased necessity of repeated revascularization at 1 year [16-18]. Kim et al. observed more frequent occurrence of the composite end point at 12-month follow-up in the single-vessel than the multivessel $\mathrm{PCl}$ group, mainly due to higher rates of cardiac deaths and recurrent MI [19]. Due to high diversity of patients with multivessel CAD presenting with NSTE-ACS it is worth taking steps towards selection of patients who would benefit the most from a complete revascularization strategy.

As presented, the main factors influencing choice of treatment strategy were anatomic limitations (primarily CTO), greater number of treated vessels and worse clinical conditions, which led to incomplete revascularization during the index hospital stay. The results of our analysis were similar to the study conducted by Head et al. in the SYNTAX trial population, which demonstrated that the independent predictors of IR-PCI were hyperlipidemia, total occlusions and number of significant lesions [11]. Moreover, Brener et al. observed a significant association between implementation of culprit-vessel $\mathrm{PCl}$ with occurrence of chronic kidney disease, prior $\mathrm{PCl}$, peripheral artery disease, older age of patient, smoking and low left ventricular ejection fraction. However, in patients with heart failure operators more frequently decided to conduct multivessel $\mathrm{PCI}$ [21]. In our study the main cause of incomplete revascularization was occurrence of CTO, which concerned almost $70 \%$ of patients in the IR-PCI group. Patients scheduled for percutaneous intervention or cardiac surgery after discharge were excluded from our analysis; therefore, it may be assumed that the operator did not anticipate intervention within CTO in the future. It has been demonstrated that CTO is an independent factor of adverse prognosis in patients with NSTE-ACS [34]. In view of these data, it appears that the presence of CTO in the discussed population of patients is one of the most demanding clinical scenarios and requires careful planning of the treatment strategy including consideration of performing CABG or percutaneous CTO recanalization. Nonetheless, in our study multivariate analysis demonstrated that $\mathrm{CR}-\mathrm{PCl}$ regardless of the presence of CTO is related to a favorable long-term prognosis.

The most important limitation of our study is the relatively small numbers of patients in each group. In addition, the retrospective nature of the study and the related consequences (selection biases) makes it difficult to generalize the conclusions of the present results. Multivariate analysis models may be biased because of the potential effect of confounding predictors that were not accessible. The decision of an invasive treatment strategy was dependent on the opinion of the operator or heart team, and could change during the period of hospitalization. Another important limitation was the method of angiographic data collection. The severity and location of coronary lesions were based on visual examination of the operator or quantitative analysis without functional assessment or available data of the SYNTAX or residual SYNTAX score. It is also assumed that the first treated artery was the culprit vessel. The results of multivariate analysis may be biased due to the potential impact of factors that were not available in our database.

Due to the observational design of the present study and despite the use of advanced adjustment methods, potential selection biases could have occurred. Multivariate analysis and propensity score-matching models may be biased because of the potential effect of confounding predictors that were not accessible. Coronary angiographic analysis was based on visual estimation with QCA, without available data of the SYNTAX score.

\section{Conclusions}

Completeness of revascularization in patients with NSTE-ACS and multivessel CAD depends on the complexity of coronary disease and individual risk profile and regards a minority of patients. CR-PCI is an independent predictor of improved 12-month outcomes and therefore should be considered when it is feasible. However, CR-PCI is not independently associated with 12-month mortality. The findings of this single-center observational study may not be generalizable for the overall NSTE-ACS population and are only hypothesis-generating.

\section{Conflict of interest}

The authors declare no conflict of interest.

\section{References}

1. Hamm C, Bassand JP, Agewall S, et al. ESC Guidelines for the management of acute coronary syndromes in patients presenting without persistent ST-segment elevation. The Task Force for the management of acute coronary syndromes (ACS) in patients presenting without persistent ST-segment elevation of the European Society of Cardiology (ESC). Eur Heart J 2011; 32: 2999-3054.

2. Windecker S, Kolh P, Alfonso F, et al. 2014 ESC/EACTS Guidelines on myocardial revascularization: The Task Force on Myocardial Revascularization of the European Society of Cardiology (ESC) and the European Association for Cardio-Thoracic Surgery (EACTS) Developed with the special contribution of the European Association of Percutaneous Cardiovascular Interventions (EAPCI). Eur Heart J 2014; 35: 2541-619.

3. Mehta SR, Cannon CP, Fox KA, et al. Routine vs selective invasive strategies in patients with acute coronary syndromes: a collaborative meta-analysis of randomized trials. JAMA 2005; 293: 2908-17.

4. Neumann FJ, Kastrati A, Pogatsa-Murray G, et al. Evaluation of prolonged antithrombotic pretreatment ('cooling-off' strategy) before intervention in patients with unstable coronary syndromes: a randomized controlled trial. JAMA 2003; 290: 1593-9.

5. Rasoul S, Ottervanger JP, de Boer MJ, et al. A comparison of dual vs. triple antiplatelet therapy in patients with non-ST-segment elevation acute coronary syndrome: results of the ELISA-2 trial. Eur Heart J 2006; 27: 1401-7. 
6. Montalescot G, Cayla G, Collet JP, et al. Immediate vs delayed intervention for acute coronary syndromes: a randomized clinical trial. JAMA 2009; 302: 947-54.

7. Lansky JA, Goto K, Cristea E, et al. Clinical and angiographic predictors of short- and long-term ischemic events in acute coronary syndromes: results from the Acute Catheterization and Urgent Intervention Triage strategY (ACUITY) Trial. Circ Cardiovasc Interv 2010; 3: 308-16.

8. Beigel R, Matetzky S, Gavrielov-Yusim N, et al. Predictors of highrisk angiographic findings in patients with non-ST-segment elevation acute coronary syndrome. Catheter Cardiovasc Interv 2014; 83: 677-83.

9. Palmer ND, Causer JP, Ramsdale DR, et al. Effect of completeness of revascularization on clinical outcome in patients with multivessel disease presenting with unstable angina who undergo percutaneous coronary intervention. J Invasive Cardiol 2004; 16: 185-8.

10. Rosner GF, Kirtane AJ, Genereux P, et al. Impact of the presence and extent of incomplete angiographic revascularization after percutaneous coronary intervention in acute coronary syndromes: the Acute Catheterization and Urgent Intervention Triage Strategy (ACUITY) trial. Circulation 2012; 125: 2613-20.

11. Head SJ, Mack MJ, Holmes DR Jr, et al. Incidence, predictors and outcomes of incomplete revascularization after percutaneous coronary intervention and coronary artery bypass grafting: a subgroup analysis of 3-year SYNTAX data. Eur J Cardiothorac Surg 2012; 41: 535-41.

12. van den Brand MJ, Rensing BJ, Morel MA, et al. The effect of completeness of revascularization on event-free survival at one year in the ARTS trial. J Am Coll Cardiol 2002; 39: 559-64.

13. Hannan EL, Racz M, Holmes DR, et al. Impact of completeness of percutaneous coronary intervention revascularization on longterm outcomes in the stent era. Circulation 2006; 113: 2406-12.

14. Goldstein JA, Demetriou D, Grines CL, et al. Multiple complex coronary plaques in patients with acute myocardial infarction. N Engl J Med 2000; 343: 915-22.

15. Asakura M, Ueda Y, Yamaguchi O, et al. Extensive development of vulnerable plaques as a pan-coronary process in patients with myocardial infarction: an angioscopic study. J Am Coll Cardiol 2001; 37: 1284-8.

16. Shishehbor $M H$, Lauer MS, Singh IM, et al. In unstable angina or non-ST-segment acute coronary syndrome, should patients with multivessel coronary artery disease undergo multivessel or culprit-only stenting? J Am Coll Cardiol 2007; 49: 849-54.

17. Zapata GO, Lasave LI, Kozak F, et al. Culprit-only or multivessel percutaneous coronary stenting in patients with non-ST-segment elevation acute coronary syndrome: one-year follow-up. J Interv Cardiol 2009; 22: 329-35.

18. Lee HJ, Song YB, Hahn JY, et al. Multivessel vs single-vessel revascularization in patients with non-ST-segment elevation acute coronary syndrome and multivessel disease in the drug-eluting stent era. Clin Cardiol 2011; 34: 160-5.

19. Kim MC, Jeong MH, Ahn Y, et al. What is optimal revascularization strategy in patients with multivessel coronary artery disease in non-ST-elevation myocardial infarction? Multivessel or culprit-only revascularization. Int J Cardiol 2011; 153: 148-53.

20. Hassanin A, Brener SJ, Lansky AJ, et al. Prognostic impact of multivessel versus culprit vessel only percutaneous intervention for patients with multivessel coronary artery disease presenting with acute coronary syndrome. Eurointervention 2015; 11 : 293-300.
21. Brener SJ, Milford-Beland S, Roe MT, et al. Culprit-only or multivessel revascularization in patients with acute coronary syndrome: an American College of Cardiology National Cardiovascular Database Registry report. Am Heart J 2008; 155: 140-6.

22. Bauer T, Zeymer U, Hochadel M, et al. Prima-vista multi-vessel percutaneous coronary intervention in haemodynamically stable patients with acute coronary syndromes: analysis of over 4.400 patients in the EHS-PCI registry. Int J Cardiol 2013; 166: 596-600.

23. Onuma Y, Muramatsu T, Girasis C, et al. Single-vessel or multivessel $\mathrm{PCl}$ in patients with multivessel disease presenting with non-ST-elevation acute coronary syndromes. Eurointervention 2013; 9: 916-22.

24. Thygesen K, Alpert JS, White HD, Joint ESC/ACCF/AHA/WHF Task Force for the Redefinition of Myocardial Infarction. Universal definition of myocardial infarction. Joint ESC/ACCF/AHA/WHF Task Force for the Redefinition of Myocardial Infarction. Eur Heart J 2007; 28: 2525-38.

25. Levine GN, Bates ER, Blankenship JC, et al. American College of Cardiology Foundation; American Heart Association Task Force on Practice Guidelines; Society for Cardiovascular Angiography and Interventions. 2011 ACCF/AHA/SCAI Guideline for Percutaneous Coronary Intervention. J Am Coll Cardiol 2011; 58: e44-122.

26. Ong ATL, Serruys PW. Complete revascularization: coronary artery bypass graft surgery versus percutaneous coronary intervention. Circulation 2006; 114: 249-55.

27. de Bruyne B. Multivessel disease: from reasonably incomplete to functionally complete revascularization. Circulation 2012; 125 : 2557-9.

28. Cutlip DE, Windecker S, Mehran R; the Academic Research Consortium. Clinical end points in coronary stent trials a case for standardized definitions. Circulation 2007; 115: 2344-51.

29. Kidney Disease: Improving Global Outcomes (KDIGO) Acute Kidney Injury Work Group. KDIGO Clinical Practice Guideline for Acute Kidney Injury. Kidney Inter Suppl 2012; 2: 1-138.

30. Shaw LJ, Berman DS, Maron DJ, et al. Optimal medical therapy with or without percutaneous coronary intervention to reduce ischemic burden: results from the Clinical Outcomes Utilizing Revascularization and Aggressive Drug Evaluation (COURAGE) trial nuclear substudy. Circulation 2008; 117: 1283-91.

31. Hannan EL, Wu C, Walford G, et al. Incomplete revascularization in the era of drug-eluting stents: impact on adverse outcomes. J Am Coll Cardiol Intv 2009; 2: 17-25.

32. Gössl M, Faxon DP, Bell MR, et al. Complete versus incomplete revascularization with coronary artery bypass graft or percutaneous intervention in stable coronary artery disease. Circ Cardiovasc Interv 2012; 5: 597-604.

33. Hochman JS, Sleeper LA, Webb JG, et al. Early revascularization and long-term survival in cardiogenic shock complicating acute myocardial infarction. JAMA 2006; 295: 2511-5.

34. Gierlotka M, Tajstra M, Gąsior M, et al. Impact of chronic total occlusion artery on 12-month mortality in patients with nonST-segment elevation myocardial infarction treated by percutaneous coronary intervention (From the PL-ACS Registry). Int J Cardiol 2013; 168: 250-4. 